'Position exchange: The social development of agency' by Alex Gillespie in New Ideas in Psychology, April 2012, Volume 30, Issue 1, Pages 32-46. doi:10.1016/j.newideapsych.2010.03.004.

This is the peer reviewed version of this article.

NOTICE: this is the author's version of a work that was accepted for publication in New Ideas in Psychology. Changes resulting from the publishing process, such as peer review, editing, corrections, structural formatting, and other quality control mechanisms may not be reflected in this document. Changes may have been made to this work since it was submitted for publication. A definitive version was subsequently published in New Ideas in Psychology, VOL 30, ISSUE 1, (April 2012). DOI: 10.1016/j.newideapsych.2010.03.004. 
Position Exchange: The Social Development of Agency

\author{
Alex Gillespie \\ Department of Psychology \\ University of Stirling \\ Stirling FK9 4LA \\ UK
}

alex.gillespie@stir.ac.uk 
Position Exchange: The Social Development of Agency

\begin{abstract}
Human agency can be defined in terms of acting independently of the immediate situation. Humans have a considerable independence from immediate situational demands because, on the one hand, they are able to distanciate from ongoing activity and reflect upon it, while on the other hand, they are able to identify with other people in different situations. It is argued that this form of agency arises through intersubjectivity because intersubjectivity enables the actor to take a perspective outside of the immediate situation and thus extricating the actor from the immediate situation. The paper contributes to the question of how intersubjectivity, as the basis of agency, develops. Explanations from phenomenology, child development and mirror neuron research are critically reviewed and the novel idea of position exchange is advanced. The paper concludes by examining some of the implications of position exchange for our understanding of the development of agency focusing upon mirror neurons, role play and autism.
\end{abstract}

Key words: Agency, position exchange, Mead, intersubjectivity, mirror neurons, dolls 


\section{Position Exchange: The Social Development of Agency}

Human agency can be defined as the degree to which an agent can act independently of the immediate situation. This sociocultural definition, which stems from Dewey and Mead, emphasises not only the power to act, but the degree to which an action is motivated by concerns originating outside of the immediate situation. The immediate situation is the here-and-now perceptual and experiential situation which arises as a function of the agent's immediate impulses combined with situational affordances, demands and constraints. The organism without agency is compelled to act by stimuli in the immediate situation. But, the organism with a degree of agency stands apart from the immediate situation and can be motivated by concerns beyond the situation, such as a distant goal, an abstract principle, or concern for someone else. This narrow definition shares much with the developmental concept of psychological distancing, as theorised by Piaget in terms of decentration and Vygotsky in terms of semiotic mediation.

Köhler's (1925/1999) classic research on problem solving amongst apes reveals the extent to which apes are 'trapped' within the immediate perceptual situation. Köhler, when introducing bananas into the apes' cages, noted how the gaze of the apes was transfixed by the desirable stimuli. In the language of behaviourism, the apes were under stimulus control. This observation prompted Köhler to suspend the bananas up high and out of reach. Initially the apes lunged up in vain. Only with practice did the apes learn to swipe down the desirable cache using a stick. Köhler 
presents such problem solving as instances of insight and agency. However, he also emphasises that this agency is limited:

Even sticks that have already been used often both by Tschego and Koko [two apes] seem to lose all their functional or instrumental value, if they are at some distance from the critical point. More precisely: if the experimenter takes care that the stick is not visible to the animal when gazing directly at the objective and that, vice versa, a direct look at the stick excludes the whole region of the objective from the field of vision - then, generally speaking, recourse to this instrument is either prevented or, at least, greatly retarded, even when it has already been frequently used. (Köhler, 1925/1999, p. 38)

Problems, such as obtaining the bananas, could be solved by the apes, provided that all the parts of the solution were presented within the perceptual field simultaneously. If the ape had to turn around to see the stick, then in turning away from the bananas, the stimuli of the bananas faded and thus the relevance of the stick seemed to disappear. Köhler's apes were, to a large extent, trapped within their immediate perceptual field.

It is too simplistic to say that apes, and other non-human animals, are slave to the immediate situation while humans are independent. Köhler and others have reported instances when apes do seem able to break away from stimulus control, in some cases even running off to find a tool which can be used to solve the given problem. Equally, studies of addiction and habit clearly demonstrate that humans are often enslaved by situational stimuli. Situations for humans are often social. Classic research in social psychology demonstrates the surprising extent to which human behaviour can be determined by immediate social demands, such as authority (Milgram, 1969), conformity (Asch, 1951) and role expectation (Haney, Banks, \& Zimbardo, 1973). Yet, despite often being enslaved by the situation, human's relation to the situation, when compared to non-human primates, is peculiar.

Consider the subjects in Milgram's (1969) experiments on obedience to authority who believed that their actions were causing distress. The fact that the 
majority continued to obey the authority demonstrates the power of the situation, and in this sense we could say subjects lacked agency. Yet, close analysis of the transcripts and observations reveals that the subjects, despite carrying out the behaviour, resisted it, disagreed with it, and spoke out against it. Subjects made appeals on behalf of the seemingly distressed victim and they demanded that the experimenter take responsibility. In other words, while the subjects' behaviour was perhaps trapped by the situation, the subjects' thoughts were not. Their thoughts were filled with questions about their behaviour, reflecting upon it from external points of view, such as the perspective of the victim and experimenter, but also from ethical and religious points of view. And the subjects' thoughts were also with the victim, feeling for and identifying with the victim's apparent distress. While subjects' behaviour was bound by the situation, their thoughts were moving between the perspectives of the victim, the experimenter, and more general potential audiences. For the purpose of this paper, these movements of thought beyond the immediate situation are assumed to be the basis of agency. That is to say, human agency is conceptualised as a form of psychological distancing (Sigel, 2002). Building on this conceptualisation, the paper will advance a theory of how human agency develops.

The aim of the present paper is to theorise how the ability to transcend the immediate here-and-now situation develops. First, the paper will distinguish two processes of extrication from the immediate situation, namely, distanciation and identification. Second, it will argue that central to these two processes is intersubjectivity. Specifically, perspective taking enables people to reflect upon their own situation and participate in the situations of others. Third, the paper will review theories about how intersubjectivity develops. The key issue is the correspondence problem, namely, how $1^{\text {st }}$ and $3^{\text {rd }}$ person perspectives become integrated to such an 
extent as to form a rich fabric of intersubjectivity with enables perspective taking? Fourth, the paper proposes position exchange as a novel theory to explain the formation of intersubjectivity. Finally, the paper concludes by considering the implications of this new theory for the development of agency.

\section{Participation and Identification}

Two distinct processes of extrication from the immediate situation can be distinguished, called distanciation and identification. This distinction reoccurs in diverse contexts. It corresponds closely to Adam Smith's (1759) distinction between the respectable virtues which lead to self-regulation through the eyes of others and the amiable virtues which lead to empathy. It also corresponds closely to Ricoeur's (1973) methodological advice to move between distanciation and participation when doing hermeneutics. In both cases the distinction is between, on the one hand, distanciation from and reflecting upon self, and, on the other hand, identifying with, empathising with, or participating in the feelings and experience of someone else. The distinction also occurs in film studies (e.g., Grodal, 1997), but while they use identification in the same way (i.e., empathetic engagement with the characters) they use distanciation to mean disengagement from the film, not distanciation from self leading to selfreflection. Let us examine these two dynamics more closely.

Distanciation refers to an actor 'stepping out' of ongoing action by reflecting upon one's self and mediating ongoing activity. This phenomenon of self-mediation has been much studied in cultural psychology. Vygotsky and Luria (1930/1994) provide an early example. They observe that young children often talk themselves through complex problems and suggest that the children use egocentric speech to 
distanciate themselves from the immediate situation, and to direct their own behaviour. The children ask themselves questions about the task, much as a parent might, and then proceed to provide the answers. The existence of such self-talk has been widely corroborated, and research has shown that inhibiting children from egocentric speech is detrimental to their problem solving performance (Winsler \& Naglieri, 2003).

Valsiner (2000) describes the process of distanciation in terms of semiotic mediation. In the children's self-talk, for example, the words spoken build up a semiotic structure that provides a cognitive platform outside of the immediate situation which the child then uses to direct their behaviour within the immediate situation.

The person becomes simultaneously an actor, who is immersed in the given 'situated activity context', and a reflexive agent who is distanced from the very setting in which he or she is immersed. This duality is relevant for transcending the adaptational demands of the here-and-now context, and guides the development towards increasing autonomy. (Valsiner, 2000, p. 50)

In studies from participants deciding whether to pretend to shoot an image of the Ku Klux Klan (Valsiner, 2003) to philosophers working out a philosophical argument (Gillespie, 2006a), research shows how people use semiotic forms (discourses, cultural meanings, common sense, social representations, etc.) in order to take up an external position and reflect upon themselves. In this sense, cultural semiotic forms are symbolic resources which actors use to distanciate from the hereand-now and to engage in reflection (Zittoun, Duveen, Gillespie, Ivinson, \& Psaltis, 2003).

Clearly there is something distinctive about symbols and semiotic forms which enables distanciation. Although there is uncertainty about what this distinctive feature is, one line of theory coming from Mead and Vygotsky is that symbols enable distanciation because they contain within themselves the perspectives of others (Zittoun, Gillespie, Cornish, \& Psaltis, 2007). That is to say, symbols are 
fundamentally intersubjective (Gillespie, in press). Thus, just as speaking entails taking the perspective of one's audience (Rommetveit, 1974), so speaking to oneself entails speaking from a perspective beyond one's immediate situation. Language, and semiotic and symbolic forms generally, form a system of meanings that transcend specific immediate here-and-now contexts thus enabling distanciation. But this only leads to the question of how this transcendent intersubjective structure is constructed. Before addressing this question, let us consider the second dynamic of agency, namely, participation.

The second process that constitutes human agency is the peculiarly human tendency to vicariously identify with, or participate in, the actions and experiences of others. Identification, again the basis of Smith's (1759) amiable virtues, is most evident in empathy, sympathy, einfühlung, altruism, and the vicarious experiencing of an other's emotion (Hatfield, Cacioppo, \& Rapson, 1994).

Identification, as a dimension of agency, in clearly evident in Milgram's research mentioned above. The subjects who resisted the authority of the experimenter and refused to continue, did so by appealing to the situation of the victim. Even the subjects who did obey made regular protests to the experimenter about the situation of the victim. These appeals reveal a degree of identification with the victim. The subjects felt for the victim and were motivated to act on behalf of the victim. In terms of our definition of agency, such action is particularly agentic because not only does it demonstrate a significant degree of independence from ones' own immediate situation (i.e., the demand of authority) but it reveals a motivation to act on the basis of someone else's immediate situation, even when that situation conflicts with the demands of one's own situation (i.e., the demand of authority). 
Identification as a dynamic of agency is closely related to altruism and brings to the foreground one of the etymological roots of agency, namely, to be an agent for someone and to act on their behalf. Ironically, although agents may seem to have little agency, because they act according to the wishes of another, they actually reveal the utmost agency by virtue of subordinating their own impulses and situational demands to the wishes and situational demands of another. Acting for another entails an intersubjective understanding of the perspective of the other. Indeed it entails acting more on the basis of their perspective than on the basis of one's own.

Both distanciation and identification imply intersubjectivity in general and perspective taking in particular. Milgram's subjects distanced themselves from their actions by taking the perspective of the experimenter, the victim, or a more general audience towards themselves. And even when using an abstract ethical principle to reflect upon their own action, these subjects can be conceptualised as escaping their own immediate perspective, which is embedded within the situation of authority, by taking a more general or abstract perspective. Equally, Milgram's subjects identifying with the victim, and making appeals on behalf of the victim, and even sometimes refusing to continue because of the supposed plight of the victim, entails perspective taking. In both cases, whether distancing from themselves or identifying with the other, subjects were extricated from their own immediate perspective, which was embedded in the situation of authority, by perspective taking. Yet these two dynamics of perspective taking, although complementary, are not equivalent. Distanciation entails a movement out of ones own situation to reflect upon one's own situation (i.e., self-focus). While identification entails a movement out of one's own situation to an empathetic participation in the situation of someone else (i.e., other-focus). 


\section{Agency Through Intersubjectivity}

What does it mean to say that both distanciation and participation arise through intersubjectivity? Each actor has a perspective and in so far as there is intersubjectivity, there is an integration of perspectives, such that actors can move between their own and other actors' perspectives. Intersubjectivity does not refer to having the same or a shared perspective (Matusov, 1996), rather it entails maintaining one's own perspective while also taking an alternative and distinct perspective (Marková, 2003). Perspectives are the more or less generalised. They can belong to specific situations, specific people, or to general types of people or groups. Indeed, at the most abstract level there are conceptual, ethical, and philosophical perspectives. G. H. Mead (1922) called the integration of perspectives 'the generalized other' and Rommetveit (1974) called it 'the architecture of intersubjectivity.' More recently Barresi and Moore (1996) have called it the 'intentional schema'. But in each case, this is the structure which enables perspective taking in relation to specific others (e.g., family and friends), general others (e.g., teachers, bus conductors, and judges), and abstract contexts (e.g., dealing with a complex problem or writing a theoretical paper for a broad audience).

While there can be little doubt that humans are embedded within a fabric of intersubjectivity, there is considerable debate about the precise nature and structure of intersubjectivity (Shrauger \& Schoeneman, 1979; Lundgren, 2004). Critiques have implied that intersubjectivity implies some mystical process of "mind reading" (Gergen, 1999, p. 125) which enables taking the actual perspective of others, i.e., to actually experience what another person is experiencing. Such a simplistic conception of intersubjectivity will fail because a perspective is an experience and one cannot 
'take' someone's experience. What can occur, however, is that one can be led, either by the situation or by imagination, to have an experience which is more or less similar. That is to say, even when 'taking' the perspective of another, one never actually leaves the domain of one's own experience (Epley, Keysar, Van Boven, \& Gilovitch, 2004; Gillespie, 2006b). This is in part what Mead meant by the term generalised other, namely, that one's experience is generalised to the other.

Barresi and Moore (1996) provide a particularly clear account of the structure and development of intersubjectivity, which they call the intentional schema. They propose two qualitatively distinct modes of representing intentional action. There are representations which derive from a $1^{\text {st }}$ person perspective (e.g., acting directly) and there are representations which derive from a $3^{\text {rd }}$ person perspective (e.g., observing someone else act). In lower animals, they suggest, these two representational modes remain unintegrated. Thus, for example, a frog would have some representation of other frogs (a $3^{\text {rd }}$ person perspective) and if not a representation, or at least sense of its own agency (a 1st person perspective) (see Gallagher, THIS VOLUME, for a discussion of the complexity of this sense of agency). However, the frog would not integrate these representations so as to either be able to see self from a $3^{\text {rd }}$ person perspective (i.e., distanciate) or to identify with the $1^{\text {st }}$ person perspective of another frog. There is no intersubjectivity. In higher primates, Barresi and Moore speculate, there seems to be some rudimentary and context specific integration of perspectives, but only in humans is this integration extensive and relatively context independent.

The interesting thing about Barresi and Moore's model is how well it fits with the concepts of distanciation and identification. Specifically, we can conceptualise distanciation as moving from a $1^{\text {st }}$ person point of view to a $3^{\text {rd }}$ person point of view (i.e. self-to-other]. For example, acting impulsively and eating the last chocolate might 
give way to reflecting upon that action from the standpoint of others and recognising it as selfish. Identification, on the other hand, can be conceptualised as a movement from a $3^{\text {rd }}$ person point of view to a $1^{\text {st }}$ person point of view (i.e., other-to-self). For example, upon viewing or imagining the suffering of others many people are motivated to act as an agent for others. The point is that conceptualising intersubjectivity as an integration of $1^{\text {st }}$ and $3^{\text {rd }}$ person perspectives provides a theoretical framework for understanding the dynamics of agency in general and distanciation and identification in particular.

Support for this conceptualisation of intersubjectivity comes from the discovery of mirror neurons. Mirror neurons were originally discovered in monkeys (Rizzolatti, Fadiga, Gallese, \& Fogassi, 1996). The interesting thing about these neurons is that they fired when the monkey engaged in a specific action but also when the monkey observed another monkey or human engage in the same action. Actions for which mirror neurons have been found in monkeys include, opening a nut, tearing paper, certain gestures and movements and eating related behaviours. While in monkeys the range of actions covered by mirror neurons is relatively narrow, in humans the range of actions is much more extensive (Rizzolatti \& Craighero, 2004). Given the nature of mirror neurons and the extent to which they occur in humans there has been much enthusiastic speculation about their significance. Mirror neurons have been introduced into discussions about language, theory of mind, empathy, imitation, perspective-taking, understanding, self-consciousness and agency. Some have even suggested that mirror neurons will be do for psychology what DNA has done for biology (Ramachandran, 2000).

The relevance of mirror neurons for the present discussion of agency is that they correspond very closely to the idea that intersubjectivity is an integration of $1^{\text {st }}$ 
and $3^{\text {rd }}$ person perspectives. The $1^{\text {st }}$ person proprioceptive feeling of opening a nut is quite different from the $3^{\text {rd }}$ person perspective of observing someone else do this. The feeling of opening a nut is tactile, entails muscle use, and is visually close, while the observation of someone opening a nut is perceptually distant and without motor or tactile components. Yet mirror neurons for nut opening reveal that somehow these two divergent experiences have become paired or integrated at a neurological level. Introducing the concepts of distanciation and identification at this point might help us move from mirror neurons, through the intentional schema to agency. Can this integration lead a person who is opening a nut to distanciate and reflect upon that act from the point of view of an observer? Might such an integration of perspectives underlie agentic behaviour such as resisting to open the nut (i.e., breaking out of the immediate situation and the desire for the nut) in order to avoid being seen to have a nut? Broadening the speculation, might the mirror neuron system be the neurological correlate of intersubjectivity, and thus the structure through which distanciation and identification is possible?

While speculation is tempting, we must be cautious moving from the level of mirror neurons to social relations. We know that mirror neurons are insufficient to account for imitation let alone language, otherwise macaque monkeys would imitate and use language. The evidence now suggests that mirror neurons are necessary but not sufficient for agency and self-consciousness (Decety \& Grèzes, 2006). Specifically, mirror neurons seem to produce an intersubjective resonance or cofeeling, but it is only when this is combined with the ability to also differentiate self from other that the dynamics of distanciation and identification can occur. Presumably it is this later capacity which is largely lacking in non-human primates (Barresi \& Moore, in press). 


\section{The Correspondence Problem}

The argument so far, that agency arises out of intersubjectivity, only replaces one problem with another. How does intersubjectivity develop? Or more specifically, how do $1^{\text {st }}$ person perspectives become integrated with $3^{\text {rd }}$ person perspectives? This problem, known variously as the correspondence problem or self-other matching problem, has been described as a "central scientific puzzle" (Whiten, 2005, p. 220) by many theorists.

In order to appreciate the scale of this puzzle one needs to recognise that actors with $1^{\text {st }}$ person perspectives do not inhabit the same perceptual or social field as observers with $3^{\text {rd }}$ person perspectives. The stimuli demanding responses from the actor are quite different to those that confront the observer. Consider mirror neurons for opening a nut. The proprioceptive experience of opening a nut is quite different from the perceptual experience of someone else opening a nut. The question is, how can these two very different experiences become integrated? Or consider the case of imitation. How is the visual perception of another's action translated into the same motor pattern by the observer? Specifically, consider a reciprocal facial gesture: How does the observer reciprocate a facial gesture when they only have visual access to the other's facial gesture and not to their own? How does the motor system "know" which pattern of muscle enervation will lead to the observed behaviour? Or lastly, consider the case of empathising with someone who seems to receive an electric shock, as in Milgram's experiment. Although such empathy is spontaneous, we need to ask how it is that the sight of someone else in distress can cause us to feel empathetic distress, particularly given that the sight of someone else in distress is a completely different 
situation and experience to actually being in distress. Again we are faced with the same problem of translation: how have the $1^{\text {st }}$ and $3^{\text {rd }}$ person perspectives, which are so different, become integrated?

Given the fundamental nature of this problem it is unsurprising to find that it arises in the literature on mirror neurons, imitation, child development, phenomenology, and role-taking. Each field of research has offered up possible solutions. These possible solutions can be distilled into four basic types, which are critically reviewed in the following four sub-sections.

\section{Analogical Apperception}

Edmund Husserl was one of the first to grapple with the correspondence problem. Husserl (1931) was influenced by Descartes and attempted to build his science of phenomenology on Cartesian foundations. However, he was dissatisfied with the idea of the solipsistic ego. Although he agreed with Descartes that the individual knowing mind could and should be at the centre of knowledge, he was concerned that this neglected the interconnectedness amongst people. Husserl's phenomenological analyses led him to the view that we live in a collectively created lifeworld of shared meanings. In grappling with the lifeworld, Husserl articulated the problem of intersubjectivity and raised the question of how it formed.

Husserl (1931) argues that individual subjectivities form intersubjective relations through 'analogical apperception.' Apperception means perceiving more than is perceptually given and the source of this 'more' comes from attributing analogous self experience to the other. Third person perspective knowledge of the other comes directly from perception, but the $1^{\text {st }}$ person understanding of the other's experience must come via analogy to one's own experience. The perceiver must pair their own $1^{\text {st }}$ 
person experience with the $3^{\text {rd }}$ person perception of the other. Husserl maintained that this process of pairing is a non-conscious inference that is embodied and immediate. The mechanism for this pairing is the similarity between self's experience and the experience of the other.

According to Schutz (1970), Husserl failed to fully grasp the correspondence problem which entails an asymmetry between one's own experience and the experience of the others' experience. The same experience looks very different depending on whether it is experienced outside-in or inside-out, and thus the 'similarity' is not obvious. Schutz writes:

The other body is visually perceived, but my body is not, as a rule, visually perceived by me, and even if it is, then only partially. My living body is, to be sure, always present and given as the primal instituting organ. But it is present as inner perception of its boundaries and through the kinaesthetic experience of its functioning. It is thus present precisely in a way which is as dissimilar as possible from the external perception of an animate body other than mine and therefore can never lead to an analogical apperception. (Schutz, 1970, p. 63)

In this excerpt, Schutz articulates the correspondence problem clearly and why Husserl's concept of similarity is not going to solve the problem. When discussing the problem, Schutz speculates about a possible role for mirrors. Mirrors provide the actor with both $1^{\text {st }}$ and $3^{\text {rd }}$ person points of view simultaneously, thus enabling an association or integration of these perspectives. But, Schutz argues, mirrors are not up to the task. Although mirrors are commonplace today, they were previously very rare being produced out of polished metal surfaces, yet there is no evidence to suggest that the widespread introduction of mirrors created any significant advance in intersubjectivity.

\section{Innate Capacity}

Research on infant imitation has been widespread since the work of James Mark Baldwin. While Baldwin emphasised joint activity, more recent research has 
reduced imitation to an innate cognitive capacity (Müller \& Runions, 2003). In support of such an innate capacity, Meltzoff and Moore (1983) have presented evidence that newborn babies can imitate several basic facial gestures. This imitation has been observed before there has been any opportunity for learning. On this basis, Meltzoff and Moore (1997) have developed the argument for an innate capacity for inter-modal mapping.

The core idea is that imitation is a matching-to-target process in which a proprioceptive feedback loop enables imitators to monitor performance and provide continuous correction relative to a visual target behaviour. Such monitoring is possible because human actions are assumed to be coded in a "supramodal act space" which is not modality-specific (Meltzoff, 2005, p. 72). Because this level of representation is not modality-specific, occurring at a higher level of abstraction, both motor information and visual information can be easily compared. The supramodal act space provides, then, trans-modal representations that transcend the immediate differences between $1^{\text {st }}$ and $3^{\text {rd }}$ person perspectives. Blending research traditions one could say that the supramodal act space, if it exists, provides evidence for Husserl's argument about necessary similarity.

The problem with the idea that there is a high level form of trans-modal representation is that it posits such a powerful cognitive capacity that it effectively side-steps the correspondence problem. Indeed, Meltzoff dismisses the problem quite explicitly when he writes:

Metaphorically, we can say that exteroception (perception of others) and proprioception (perception of self) speak the same language from birth; there is no need for 'association.' (Meltzoff, 2005, p. 72)

To say that there is no need for association, is to say that there is no problem of correspondence. All the explanation is packed into the innate supramodal act space. 
Such a move would be acceptable if the evidence warranted it, but the evidence upon which Meltzoff relies is contentious. Specifically, the behaviours imitated by neonates may be much fewer than Meltzoff claims to have found (Anisfeld, 1996). Secondly, even if Meltzoff is right about the innate existence of basic imitation, the question about how this basic imitation develops into the rich intersubjectivity that characterises humans remains to be explained (Müller \& Runions, 2003, p. 31). The theory does not provide any indication of how the expansion of this mapping can be facilitated, or why in some cases it fails to develop. Gallagher (2004) has tried to do this, but he relies heavily on interactionist theory. In sum, the innate capacity argument assumes a lot and yet still fails to account for anything but very rudimentary imitation.

\section{Concurrent Activation}

A third possible solution to the correspondence problem, arising in different literatures, relies upon traditional learning mechanisms and as such is more parsimonious than postulating an innate capacity. The core idea is that $1^{\text {st }}$ person perspective representations and $3^{\text {rd }}$ person perspective representations can become associated together through basic learning processes by virtue of being enervated simultaneously. Schutz's discussion about mirrors, mentioned above, presents an early glimmer of this idea, but since then it has been extensively elaborated.

MacCoby (1959) deals with the correspondence problem by first emphasising that many behaviours actually look the same to both actor and observer. This is especially true for actions involving the hands. For example, watching someone else tie a knot looks very similar to observing oneself tie a knot. In such a context the same brain area is enervated when acting or observing, thus enabling a translation between $1^{\text {st }}$ and $3^{\text {rd }}$ person perspectives. But what about asymmetrical correspondences such as 
facial gestures? To account for these MacCoby appeals to the fact that adults often mirror the behaviours, especially facial gestures, of their children. Such imitation creates a situation of concurrent activation. For example, the child while enacting a smile from a $1^{\text {st }}$ person perspective would also receive the $3^{\text {rd }}$ person perspective visual information of an adult imitating and smiling, and thus learn to associate the feeling of smiling with the sight of a smile. Unlike Meltzoff, this is not about children imitating adults, but about adults imitating children.

A more recent variant of the concurrent activation theory comes from Barresi and Moore (1996, p. 110) who invoke it to help account for the integration of $1^{\text {st }}$ and $3^{\text {rd }}$ person perspectives within the intentional schema. Barresi and Moore mention the use of mirrors and parental imitation, but they add to the mix a third pathway for concurrent activation. Building on their account of joint attention, if two agents are in the same situation and are both engaged in the same intentional relation with an object, then both agents will experience both $1^{\text {st }}$ and $3^{\text {rd }}$ person perspectives simultaneously. The concurrent activation of both perspectives could then form the basis for forming an association, which contributes to the development of intersubjectivity, which once consolidated enables movement between $1^{\text {st }}$ and $3^{\text {rd }}$ person perspectives even in the absence of concurrent activation. Examples of such simultaneous activities might be a child walking with their parents, or helping to carry something, or simultaneous laughing.

Perhaps the most recent manifestation of the concurrent activation theory comes from Heyes' (2005; Brass \& Heyes, 2005) account of imitation. Although she does not mention the aforementioned theorists, she hits upon the same pathways for concurrent activation, placing specific emphasis on adult imitation. And it is in response to Heyes' (2005) presentation of the concurrent activation theory that 
Whitten (2005) has raised a potentially fatal critique of the idea. Whitten argues that concurrent activation presupposes a solution to the correspondence problem by assuming that children will be able to recognise imitation. Only a minority of adult action towards children is imitation, so, how do children know when adults are imitating them? Obviously children cannot know when adults are imitating them, because that would imply that they have already overcome the correspondence problem and thus that there is no learning to be done. But if the children do not know when adults are imitating them then it seems reasonable to suppose that sometimes mistakes would be made. For example, the child might pair the feeling of going asleep, with relaxed facial muscles, with the sight of adults smiling with relief. Yet, as Whitten point out, there are no common examples of such mistaken 'integrations' of $1^{\text {st }}$ and $3^{\text {rd }}$ person perspectives. Although levelled explicitly at parental imitation, the critique also applies to the simultaneous action pathway postulated by Barresi and Moore (1996). How would the children know that the people they are with are engaging in the same activity as them? The mirror pathway is somewhat less affected by this critique because mirrors always provide imitative feedback.

A second critique of the concurrent activation idea arises from an overly narrow conception of the correspondence problem which reduces it to the problem of integrating motor response with visual perception. The problem is that there is much more to the correspondence problem than cross-modal integration. Indeed, there are often situations in which both actors confront perceptually identical stimuli, yet they are in completely different roles and thus situations. Consider the case of a face-to-face interaction between a buyer and seller. At many levels, both perceive the same perceptual stimuli, such as their immediate surrounding, the item to be purchased, the money, and the other. Yet each is in a completely different social situation with 
different motivations and goals. The difference is less about perception and more about role. In such a social situation there is 'a differentiation problem', namely how do the actors know they are in different roles despite being in the same perceptual situation? Intersubjective understanding is necessary if the exchange is to pass successfully. So how has this higher and more social level of intersubjectivity formed? Any account for the formation of intersubjectivity that limits itself to the formation of cross modal associations will fail to account for this higher and more complex, but nonetheless necessary, level of integration.

\section{Common Stimulus}

The fourth mechanism by which $1^{\text {st }}$ and $3^{\text {rd }}$ person perspectives may become integrated derives from a peculiar property of auditory stimuli and especially vocal gestures. This idea was originally put forward by Mead (1912). Mead, when discussing the correspondence problem, points out that the divergence between $1^{\text {st }}$ and $3^{\text {rd }}$ person perspectives is primarily visual, and is much less pronounced in the auditory modality. Mead writes:

While one feels but imperfectly the value of his own facial expression or bodily attitude for another, his ear reveals to him his own vocal gesture in the same form that it assumes to his neighbour. (Mead, 1912, p. 403)

On this basis, Mead speculates that the auditory modality may act as a bridge, integrating $1^{\text {st }}$ and $3^{\text {rd }}$ person perspectives. Mead ring-fences the correspondence problem as peculiar to the visual modality, and points out that the auditory modality has a different structural logic which does not create the same divergences of perspective. Just like people cannot normally see around corners, so they cannot see their own facial expressions or bodily posture. But people can hear around corners, and they can hear their own utterances. Mead's suggestion is that if the correspondence 
problem is created in the visual modality maybe it is overcome in the auditory modality.

Specifically, Mead considered vocal gestures to act as auditory bridges between $1^{\text {st }}$ and $3^{\text {rd }}$ person perspectives because vocal gestures are heard very similarly by both speakers and listeners (Farr, 1996, chapter 4). Indeed, on this basis Mead argued that reflective self-consciousness is primarily an auditory phenomenon arising when we hear ourselves speak, either externally or internally (Gillespie, 2005). Also, Mead used this to explain why language was based on vocal utterances rather than motor gesture.

Mead's ideas about the peculiarity of the auditory medium have received little attention. However, the idea, without his name, has recently been promoted by Heyes (2005). Alongside the idea of concurrent activation, discussed above, Heyes makes the case for what she calls a common stimulus. The idea is that $1^{\text {st }}$ and $3^{\text {rd }}$ person perspectives may become associated indirectly by virtue of a stimulus that is common to both perspectives. Heyes gives the following example:

For example, a child may hear the sound of a word such as "frown", sometimes when she is frowning and at other times when she sees another person frowning (Heyes, 2005, p. 160)

Although this example which Heyes gives entails a shared auditory sound, she does not restrict her version of the idea to the auditory medium. Instead she simply refers to common stimuli, suggesting that these could be visual, motor or otherwise. On the one hand Heyes' theory lacks specificity regarding the auditory medium, but on the other hand her theory is more general.

The idea of indirect associations forming via common stimuli, especially auditory stimuli, is supported by some evidence. First, Kohler et al. (2002) have found that there are mirror neurons which fire to auditory stimuli accompanying action done 
by self and action done by other. These neurons may thus act as a bridge integrating $1^{\text {st }}$ and $3^{\text {rd }}$ person perspectives. Second, Knoblich and Jordan (2002) have found that introducing an auditory stimulus in the context of joint activity under a condition of divergent visual perspectives facilitated coordination of perspectives. Finally, finegrained analysis of people's utterances reveals, as Mead speculated, that people reflectively respond to their own utterances while speaking (Gillespie, 2007).

Overall the idea of a common stimulus overcomes a number of the problems. Unlike analogical apperception and the idea of innate capacity, the idea of a common stimulus postulates a clear and parsimonious mechanism for the integration of perspectives. Unlike the idea of concurrent activation, it does not presuppose the capacity for imitation or perspective taking. However, I want to argue, that as formulated by Heyes and also to some extent by Mead, it is an incomplete theory. To fully understand how a common stimulus can lead to an integration of perspectives and thus the formation of intersubjectivity or the intentional schema, an additional concept is needed, namely, position exchange.

\section{Position Exchange}

Position exchange (Gillespie, 2005, 2006; Martin, 2006a, 2006b) is a novel possible solution to the correspondence problem. The idea assumes that social life is structured in terms of distinct yet complementary social positions. Social positions arise in social interaction by virtue of the different positions participants take vis-à-vis each other. Interactions from smiling at someone to the negotiation of a peace treaty entail social positions. Smiling at someone is an interaction which creates the social positions of smiling and being smiled at. Slightly more complex examples of social 
positions include talking/listening, buying/selling and commanding/obeying. In each social interaction, the social positions cannot be defined in isolation, rather they are always defined in part by the complementary social position. Observing the smile is the complementary social position to smiling and acquiescing or resisting are the complementary social positions for demanding.

Each social position sustains a distinctive motor and/or perceptual perspective and also often a social role, that is a set of expectations about what each partner in the interaction should do. Observing a smile often entails the role expectation of reciprocating the smile, and buying entails the role expectation of paying, and so on. Thus social positions actually contribute to the correspondence problem by virtue of creating and sustaining divergent perspectives. But in so far as social positions contribute to the problem of divergent perspectives, so movement of actors between these social positions overcomes the problem.

Position exchange assumes that social positions are relatively stable. Thus although actors cannot 'mind read' they can, by exchanging social position, come to experience the same perspectives. More specifically, if the position exchange tends to occur with particular frequency between complementary social positions, then the integration of $1^{\text {st }}$ and $3^{\text {rd }}$ person perspectives would be facilitated. The mechanism of integration is not a mental act but a social act.

Consider normal conversation and the alteration between speaking and listening. Within one conversational exchange, these positions will be exchanged numerous times. These complementary positions become further elaborated in school, where children learn to both listen and present. According to the idea of position exchange this movement between the position of speaking and listening could integrate perspectives creating intersubjectivity and thus agency. Specifically, children 
become able to regulate their behaviour as listeners (e.g., not to speak but to listen) by virtue of reflecting upon themselves from the previous experience of being a speaker, and also, they become able to regulate their behaviour as speakers (e.g., speaking clearly) by reflecting upon their own behaviour from the standpoint of previous experience of listening. In both contexts the integration of perspectives through repeated position exchange enables distanciation. Such integration could also lead to identification, for example when observing another person getting embarrassed during public speaking leads to vicarious embarrassment and squirming discomfort.

Gift exchange rituals, such as Christmas, provide a second common example of position exchange. In the traditional situation the presents are piled up around the Christmas tree. All participants gather and are in the same perceptual environment. Participants take turns in giving and receiving presents. With each turn from giving to receiving or vice-versa there is position exchange. During the ritual participants move repeatedly from the position of giving to that of receiving and back until the supply of gifts is exhausted. According to the position exchange idea, integration of the perspectives of giving and receiving enables the child to give from the standpoint of receiving, and to receive from the standpoint of giving. As this integration consolidates, children become able to be polite and thankful even for presents they do not desire. Alternative examples of position exchange, analysed elsewhere, include child feeding (Martin, 2006a) and children's games (Gillespie, 2006).

Position exchange does not rely on a mysterious cognitive capacity for either analogical apperception or intermodal matching. Like the idea of concurrent activation, it shifts the burden of explanation to learning through social interaction. But the theory of concurrent activation assumes that the social position of the developing individual is fixed, and the social and physical world 'mirrors' behaviour thus creating concurrent 
activation. However, in position exchange it is the movement of actors between social positions which weaves the fabric of intersubjectivity. Although actors cannot 'take' each other's experiential perspectives directly, they can quite literally take each other's social position and thus experience a similar perspective.

Position exchange is actually implied by the idea of the common stimulus because a common stimulus entails that the same stimulus be present in two different social positions. For the word "smile" to become associated with both the motor response of smiling and the sight of someone smiling, it follows that the person concerned must have been in both the social position of smiling and the social position of observing someone smile. Equally, for the word "shopping" to act as a bridge between the perspectives of buying and selling it is necessary that the person concerned has been in both the social positions of buying and selling, and heard that word while in both social positions. As such, position exchange works in conjunction with the idea of a common stimulus, not in opposition to it. But learning is more than making associations, and position exchange goes beyond the formation of such basic associations to enable the holistic experience of another person's situation. Also, position exchange can lead toward bicolage experiences, where the observer has not actually experienced the social position of the actor, but is able to patch together an impression of the experience of the actor by bringing together several different and sometimes analogical and even just narrative experiences, thus enabling more complex forms of perspective taking - even in the absence of direct experience.

Whiten's (2005) critique of the idea of concurrent activation, namely that it presupposes a solution to the correspondence problem, could be developed and applied to position exchange. One could ask: How does the developing child know that they are moving between complementary social positions? Sometimes children move 
between non-complementary social positions (e.g., the child may receive a gift and then eat some food and then smile), so again one could ask, why are there not arbitrary pairings? There are two possible ripostes. First, position exchange occurring repeatedly between the same social positions might in itself act as a cue signalling position exchange. Such repeated position exchange is particularly evident in many children's games such as peek-a-boo, escaping/chasing games, cops and robbers and so on. The second riposte returns to the idea of common stimuli. When moving between noncomplementary social positions there is an entire change of situation, and there are very few common stimuli. However, when position exchange occurs there is often an abundance of common stimuli (both auditory and visual) which again cue the complementary nature of the social positions.

The second critique of the concurrent activation idea, namely that reducing the problematic to an integration of motor responses and visual stimuli avoids problems of integrating social roles, does not apply to position exchange. A buyer and seller, for example, are in the same perceptual environment but in different social roles. Position exchange between these roles enables an integration of their respective perspectives. In this sense, the idea of position exchange can apply both to basic integration of motor and visual modalities, but also to more complex integration of perspectives belonging to complementary social roles.

Not only does position exchange manage to avoid the critiques levelled at the alternative suggestions, but there is also a diverse body of evidence that can be marshalled in support of the theory. This research was not designed explicitly to examine the position exchange idea, nevertheless, it can be interpreted in the light of position exchange. For example, in adults (Stryker, 1956), children (Melzoff, 2005, p. 69) and even in scrub jays (Emery \& Clayton, 2001) there is evidence that having been 
in the social position of the other facilitates an understanding of the perspective of the other. More particularly, manipulations of position exchange have shown that it can reduce racism (McGregor, 1993) and stimulate more creative problem solving (Shirouzu, Miyake, \& Masukawa, 2002).

\section{Concluding Implications}

Postulating position exchange as a central mechanism in the development of intersubjectivity, and thus agency in the form of distanciation and participation, raises new issues for research. In what follows I will explore the implications of the idea of position exchange for research on mirror neurons, role play and autism.

First, consider mirror neurons in non-human primates. It is striking how narrow the range of behaviours for which mirror neuron activation has been found and indeed the even narrower range of behaviours and perceptions which each mirror neuron responds to. Specifically, it is noticeable that many mirror neurons reported are related to feeding behaviours. Indeed, Kohler et al. (2002) report that in macaques mirror neurons for the behaviour of opening a peanut are the easiest to find. Also, at the level of behaviour the most complex forms of perspective taking behaviour in non-human primates seem to congregate around feeding behaviour (e.g., Povinelli, Nelson, \& Boysen, 1992; Emery \& Clayton, 2001). Why is feeding behaviour so well represented? Or more particularly, why is there a dearth of mirror neurons in relation to other important social actions such as mating?

Position exchange suggests an answer. Feeding behaviours entail the social positions of feeding and observing while mating entails the social positions of male 
and female. The difference is that there is frequent position exchange in relation to feeding but not in relation to mating.

Developing this line of thought leads to the proposition that there are no mirror neurons in non-human primates for behaviours which do not entail position exchange. Thus, for example, we would expect there to be no mirror neurons in regard to sexual relations because, in most species, there is no position exchange between these social roles. Additionally, we would expect there to be no mirror neurons in regard to crossspecies predator/prey relations, because again there is no position exchange. In both cases the roles, or social positions, are genetically determined and there exist no social intuitions for facilitating an exchange of positions. In both sexual relations and in predator/prey relations perspective taking would be highly beneficial, having a potentially huge impact upon propagation and survival. Yet, no such ability has evolved or developed, it is suggested, because there has been no position exchange.

Position exchange leads to a conceptualisation of agency as highly contextual and gradually learned, not an abstract or absolute ability. The theory converges with Moore's (2006) concept of "intentional islands" which refer to an early phase of the integration of $1^{\text {st }}$ and $3^{\text {rd }}$ person perspectives in which the integration only exists for specific behaviours or intentional relations within specific contexts. Within these islands, or pockets of intentional competence, there is a degree of perspective taking, and thus autonomy, but this does not generalise to other behaviours or contexts. The geography of these islands, it is suggested, will be found to correspond to those social interactions in which there is position exchange.

Second, let us turn to the implications of position exchange for role play. In role playing games children simulate a range of different social roles, or positions. Moreover, children role playing often move between the roles, thus creating an ideal 
context for position exchange and the integration of perspectives. Children in all cultures role play, but what they role play varies as a function of what adults are observed to do. Depending upon the culture, children can be observed to role play building, ploughing, child minding, cooking, driving, or hunting (Edwards, 2000). Interestingly, playing at a particular role tends to cease once the child is actually put in the given role. Might it be that young children are using role play as a form of position exchange? Are they using props to support fictional position exchange, and thus explore the roles and positions which they observe around them?

One form of role play which supports such speculation is play with dolls. There is evidence of dolls going back at least four thousand years. In the Indus valley civilisation children played with wooden dolls, in America Navajo children played with elaborately tailored dolls, in Russia children played with straw dolls, and more recently children have played with commercially produced dolls (Formanek-Brunell, 1993). In Europe dolls were initially known as 'toy babies' or just 'babies' until the $18^{\text {th }}$ century when the word 'doll', a diminutive of Dorothy, became a common term. Indeed, many dolls are even made to look like babies, which is peculiar, given that they are for children not much older than babies. The question to ask is, why are dolls, especially of babies, so captivating for young children? Might it be that playing with dolls children are trying to exchange position with their caregivers and thus try to see themselves as their caregivers see them?

Prior to playing with dolls the child was the baby, cared for by others. But with the introduction of the play doll, the social positions can be reversed. The doll takes the place of the young child, and the child takes the position of the caregiver. Harris (2000, p. 31) observes that children playing with dolls play at the complete range of parental activities associated with babies, including, putting the doll to bed, bathing, 
nappy-changing, feeding, schooling, disciplining, carrying, crying and so on. In enacting each of these social situations, the child must occupy both the position of the baby and the position of the caregiver. For example, in a play sequence the baby doll might cry, the child will feed the doll, and the doll would be seen to stop crying, but might need a nappy change, which would lead the child to change the nappy and so on. Within such a sequence the child is repeatedly moving between the position of baby and caregiver. This movement occurs in imagination but it is supported by the socialmaterial prop of the doll.

According to a position exchange interpretation playing with baby dolls facilitates the integration of perspectives within which the child herself exists. The child learns not only about the situation of the caregiver, but also about the meaning of being a baby from the standpoint of a caregiver. As this integration develops, so the child becomes able to distanciate from her own childish behaviour, by taking the perspective of the caregiver, as enacted in play, and equally, becomes able to participate, to some extent, in the trials and tribulations faced by caregivers. That is to say, play with dolls might be facilitating the agency of the young child vis-à-vis being a baby. The suggestion can be examined empirically. Are young children who play extensively with baby dolls better able to take the perspective of their caregivers? Or, more particularly, are young children who play with baby dolls better able to regulate their own behaviour from the standpoint of caregivers? Are they better able to identify with caregivers? In short, does playing with baby dolls aid in extricating the young child from the immediate situation of being a baby?

Finally, building upon what has been said about role play, we now turn to the implications of position exchange for research on autism. Barresi and Moore (1996) conceptualise autism as an incomplete integration of perspectives within the 
intentional schema. If position exchange is a means to foster such integration, then we would expect position exchange to be rare amongst children with autism. Assuming the argument made above, that role play is a form of position exchange, then there is considerable evidence. For example, according to Harris and Leevers (2000) children with autism rarely engage in role play. Indeed, an absence of role play at 18 months is predictive of autism. In contrast to these findings about children with autism, children who role play more than average are considered to be more sociable and more likable by their peers (Howes, Rubin, Ross, \& French, 1988) and be more insightful about mental states (Harris, 2000, p. 37). Particularly relevant is the finding that children with autism rarely engage in any type of role reversal (Carpenter, Tomasello, \& Striano, 2005). There is also evidence suggesting that role play at 33 months predicts understanding false beliefs at 40 months (Youngblade \& Dunn, 1995). In these studies the direction of causality is indeterminate, and many would argue that it is the autism which is causing the role play. But if the thesis about position exchange is correct, and if it is position exchange which underlies the significance of role play, then an avenue opens up for potentially fruitful interventions. Games which entail position exchange provide a relatively easy form of intervention. Games could be designed to target specific behaviours in which there are problems. Extensive position exchange, within these games, might facilitate the creation of 'intentional islands' thus either mediating the impact or onset of autism.

It is also possible that similar position exchange games could be used profitably with children who have disruptive behaviour. Disruptive behaviour can be conceptualised as a lack of self control (Kendall, 1984). From the present Meadian perspective, lack of self control is a failure to respond to oneself from the standpoint of others. It is a lack of agency in the sense that the child is unable to extricate themselves 
from their immediate behaviour and see that behaviour from the perspective of others. In such cases the problem behaviours can normally be quite precisely defined, and as such, specially designed games to target those behaviours with position exchange might foster greater intersubjectivity and thus self-control in regard to those behaviours.

In conclusion, I want to point to the central thread which underlies these potential contributions to research in mirror neurons, role play and autism, namely, intersubjectivity. Intersubjectivity, as an integration of perspectives, is what lies between position exchange and agency. Position exchange weaves the integration of perspectives that is intersubjectivity, and intersubjectivity enables distanciation and identification which are the basis of agency. Intersubjectivity enables the actor embedded in a situation to transcend their own immediate embeddedness and to either take the perspective of others upon themselves, thus mediating their own activity, or to identify with others, and thus act on their behalf. The model of agency presented is highly social, in the sense of arising out of social interaction, but it does not collapse the individual's agency into the social as so often occurs with social constructionist theories (Martin \& Sugarman, 1999). Indeed, although actors gain agency through social interaction, they subsequently posses agency to the extent that they manage to extricate themselves from those same interactions. The mechanism for this liberation is intersubjectivity and the basis of intersubjectivity, I have argued, is position exchange. 
Anisfeld, M. (1996). Only tongue protrusion modeling is matched by neonates. Developmental Review, 16, 149-161.

Asch, S. E. (1951). Effects of group pressure upon the modification and distortion of judgment. In H. Guetzkow (Ed.), Groups, leadership and men. Pittsburgh, PA: Carnegie Press.

Barresi, J., \& Moore, C. (1996). Intentional relations and social understanding. Behavioral and Brain Sciences, 19, 107-154.

Barresi, J., \& Moore, C. (in press). The neuroscience of social understanding. In J. Zlatev, T. Racine, C. Sinha, \& E. Itkonen (Eds.), The shared mind: Perspectives on intersubjectivity. Amsterdam: John Benjamins.

Brass, M., \& Heyes, C. (2005). Imitation: Is cognitive neuroscience solving the correspondence problem. Trends in Cognitive Sciences, 9, 489-495.

Carpenter, M., Tomasello, M., \& Striano, T. (2005). Role reversal imitation and language in typically developing infants and children with autism. Infancy, 8 , 253-278.

Decety, J., \& Grèzes, J. (2006). The power of simulation: Imagining one's own and other's behavior. Brain Research, 1079, 4-14.

Edwards, C. P. (2000). Children's play in cross-cultural perspective: A new look at the Six Cultures study. Cross-cultural research, 34, 318-338.

Emery, N. J., \& Clayton, N. S. (2001). Effects of experience and social context on prospective caching strategies by scrub jays. Nature, 414, 443-446.

Epley, N., Keysar, B., Van Boven, L., \& Gilovitch, T. (2004). Perspective taking as egocentric anchoring and adjustment. Journal of Personality and Social Psychology, 87, 327-339.

Farr, R. M. (1996). The roots of modern social psychology: 1874-1954. Oxford: Blackwell.

Formanek-Brunell, M. (1993). Made to Play House: Dolls and the Commercialization of American Girlhood, 1830-1930. New Haven: Yale University Press.

Gallagher, S. (2004). Understanding interpersonal problems in autism: Interaction theory as an alternative to theory of mind. Philosophy, Psychiatry, \& Psychology, 11, 199-217.

Gallagher, S. (IN PRESS). Multiple aspects in the sense of agency. New Ideas in Psychology. XXXXXXXX

Gergen, K. (1999). An invitation to social construction. . London: Sage Publications.

Gillespie, A. (2005). G.H. Mead: Theorist of the social act. Journal for the Theory of Social Behaviour, 35, 19-39.

Gillespie, A. (2006). Games and the development of perspective taking. Human Development, 49, 87-92.

Gillespie, A. (2006a). Descartes' demon: A dialogical analysis of Meditations on First Philosophy. Theory \& Psychology, 16, 761-781.

Gillespie, A. (2006b). Tourist photography and the reverse gaze. Ethos, 34, 343-366.

Gillespie, A. (2007). Collapsing self/other positions: Identification through differentiation. British Journal of Social Psychology, 46, 579-595.

Gillespie, A. (in press). The intersubjective nature of symbols. In B. Wagoner (Ed.), Symbolic transformations: Toward an interdisciplinary science of symbols. London: Routledge.

Grodal, T. K. (1997). Moving pictures: A new theory of film genres, feelings, and cognition. Oxford: Clarendon Press. 
Haney, C., Banks, C., \& Zimbardo, P. (1973). Interpersonal dynamics in a simulated prison. International Journal of Criminology and Penology, 1, 69-97.

Harris, P. L. (2000). The work of the imagination. Oxford: Blackwell Publishing.

Harris, P. L., \& Leevers, H. (2000). Pretending, imagery and self-awareness in autism. In S. Baron-Cohen, H. Tager-Flusberg, \& D. Cohen (Eds.), Understanding other minds (pp. 182-202). Oxford: Oxford University Press.

Hatfield, E., Cacioppo, J., \& Rapson, R. (1994). Emotional contagion. Cambridge: Cambridge University Press.

Heyes, C. M. (2005). Imitation by association. In S. Hurley \& N. Chater (Eds.), Perspectives on imitation: Mechanisms of imitation and imitation in animals (pp. 157-176). Cambridge, MA: MIT Press.

Howes, C., Rubin, K. H., Ross, H. S., \& French, D. C. (1988). Peer interaction of young children. Monographs of the Society for Research in Child Development, $53,1-92$.

Husserl, E. (1931). Cartesian meditations: An introduction to phenomenology. Dordrecht: Kluwer.

Knoblich, G., \& Jordan, S. (2002). The mirror system and joint action. In M. I. Stamenov \& V. Gallese (Eds.), Mirror neurons and the evolution of brain and language (p. 115-124). Amsterdam: John Benjamins Publishing Company.

Kohler, E., Keysers, C., Umilta, M., Fogassi, L., Gallese, V., \& Rizzolatti, G. (2002). Hearing sounds, understanding actions: action representation in mirror neurons. Science, 297, 846-848.

Köhler, W. (1999). The mentality of apes. London: Routledge. (Original work published 1925)

Lundgren, D. C. (2004). Social feedback and self-appraisals: Current status of the Mead-Cooley hypothesis. Symbolic Interaction, 27, 267-286.

MacCoby, E. E. (1959). Role-taking in childhood and its consequences for social learning. Child Development, 30, 239-252.

Marková, I. (2003). Constitution of the self: Intersubjectivity and dialogicality. Culture \& Psychology, 9, 249-259.

Martin, J. \& Sugarman, J. (1999). The Psychology of Human Possibility and Constraint. Albany, NY: State University of New York Press.

Martin, J. (2006a). Reinterpreting internalization and agency through GH Mead's perspectival realism. Human Development, 49, 65-86.

Martin, J. (2006b). Positions, perspectives, and persons. Human Development, 49, 9395.

Matusov, E. (1996). Intersubjectivity without agreement. Mind, Culture, and Activity, $3,25-45$.

McGregor, J. (1993). Effectiveness of role playing and antiracist teaching in reducing student prejudice. Journal of Educational Research, 86, 215-226.

Mead, G. (1922). A behavioristic account of the significant symbol. Journal of Philosophy, 19, 157-163.

Mead, G. H. (1912). The mechanism of social consciousness. The Journal of Philosophy, Psychology and Scientific Methods, 9, 401-406.

Meltzoff, A. N. (2005). Imitation and other minds: The 'like me' hypothesis. In S. Hurley \& N. Chater (Eds.), Perspectives on imitation: Imitation, human development, and culture (pp. 55-77). Cambridge, MA: MIT Press.

Meltzoff, A. N., \& Moore, M. K. (1983). Newborn infants imitate adult facial gestures. Child Development, 54, 702-709. 
Meltzoff, A. N., \& Moore, M. K. (1997). Explaining facial imitation: A theoretical model. Early Development and Parenting, 6, 179-192.

Milgram, S. (1969). Obedience to authority: An experimental view. New York: Harper Torchbooks.

Moore, C. (2006). Representing intentional relations and acting intentionally in infancy: Current insights and open questions. In I. Knoblich, M. Thornton, M. Grosjean, \& M. Shiffrar (Eds.), Human body perception from the inside out (pp. 427-442). Oxford: Oxford University Press.

Müller, U., \& Runions, K. (2003). The origins of understanding self and other: James Mark Baldwin's theory. Developmental Review, 23(1), 29-54.

Povinelli, D. J., Nelson, K. E., \& Boysen, S. T. (1992). Comprehension of role reversal in chimpanzees: Evidence of empathy? Animal behaviour, 43, 633-640.

Ramachandran, V. S. (2000). Mirror neurons and imitation learning as the driving force behind the great leap forward in human evolution. Edge Website article http://www. edge. org/3rd_culture/ramachandran/ramachandran_pl. html. (accessed 26 $6^{\text {th }}$ March 2008)

Ricoeur, P. (1973). The hermeneutical function of distanciation. Philosophy Today, 17, 129-141.

Rizzolatti, G., \& Craighero, L. (2004). The mirror-neuron system. Annual review of neuroscience, 27, 169-192.

Rizzolatti, G., Fadiga, L., Gallese, V., \& Fogassi, L. (1996). Premotor cortex and the recognition of motor actions. Cognitive Brain Research, 3, 131-141.

Rommetveit, R. (1974). On message structure: A framework for the study of language and communication. London: John Wiley \& Sons.

Schutz, A. (1970). Collected papers III: Studies in phenomenological philosophy. The Hague: Martinus Nijhoff.

Shirouzu, H., Miyake, N., \& Masukawa, H. (2002). Cognitively active externalization for situated reflection. Cognitive Science, 26, 469-501.

Shrauger, J. S., \& Schoeneman, T. J. (1979). Symbolic interactionist view of selfconcept: Through the looking glass darkly. Psychological Bulletin, 86, 549573.

Sigel, I. E. (2002). The psychological distancing model: A study of the socialization of cognition. Culture \& Psychology, 8, 189-214.

Smith, A. (1759). The theory of moral sentiments (Eds, D. D. Raphael \& A. L. Macfie). Indianapolis, IN: Liberty Fund.

Stryker, S. (1956). Relationships of married offspring and parent: A test of Mead's theory. The American Journal of Sociology, 62, 308-319.

Valsiner, J. (2000). Culture and human development. London: Sage publications.

Valsiner, J. (2003). Beyond social representations: A theory of enablement. Papers on Social Representations (www.psr.jku.at), 12, 7.1-7.16.

Vygotsky, L., \& Luria, A. (1994). Tool and symbol in child development. In The Vygotsky Reader (pp. 99-174). Oxford: Blackwell. (Original work produced 1930)

Whiten, A. (2005). The imitative correspondence problem: Solved or sidestepped? In S. Hurley \& N. Chater (Eds.), Perspectives on imitation: Mechanisms of imitation and imitation in animals (pp. 220-222). Cambridge, MA: MIT Press.

Winsler, A., \& Naglieri, J. (2003). Overt and covert verbal problem-solving strategies: Developmental trends in use, awareness and relations with task performance in children aged 5 to 17. Child Development, 74, 659-678. 
Youngblade, L. M., \& Dunn, J. (1995). Individual differences in young children's pretend play with mother and sibling: links to relationships and understanding of other people's feelings and beliefs. Child Development, 66, 1472-1492.

Zittoun, T., Duveen, G., Gillespie, A., Ivinson, G., \& Psaltis, C. (2003). The use of symbolic resources in developmental transitions. Culture \& Psychology, 9, 415-448.

Zittoun, T., Gillespie, A., Cornish, F., \& Psaltis, C. (2007). The metaphor of the triangle in theories of human development. Human Development, 50, 208-229. 